\title{
An Optimal Policy for a Manufacturer's Sales Problem Using Markov Decision Model
}

\author{
Hassan Sheikh Aisha \& Akoh David \\ Department of Mathematics and Statistics, Federal Polytechnic, Bida
}

\begin{abstract}
The success rate of any firm in the industry depends greatly on its sales output. This is achievable only when certain strategies are employed to overcome competition pressures from allied firms. We utilize the tools of Markov decision process in designing our model that improves on the quality of advertisement, research and manpower development to yield an optimal choice leading to profit maximization and cost effectiveness. Finally, we solve our model equations by the value iteration method
\end{abstract}

Keywords: markov decision process, value iteration method, optimal choice policy,

\section{Introduction}

Manufacturers are confronted with many pressures during the process of production. Amongst such are the problems of competition from allied firms, product imitation by fake manufacturers, copyright abuses, outsourcing as well as inventory problems. These problems have led to a loss of goodwill and even a total collapse of many firms over the years. In a bid to addressing these problems, many scholars have explore various techniques with much results but the numerous problems cannot be treated completely in a single article, hence our work intends to address the problems associated with competition pressures confronting manufacturers as they sell products.

\section{Some Definitions}

This section consists of definitions and theorems that relate to Markov decisions processes and optimality in decision making.

\subsection{Markov Chain:}

According to (Howard, R.A. 1960) Markov chain is a discrete time process governed by a discrete state space, $\mathrm{E}$ (observed at discrete time points) and transition matrix $\mathrm{P}$, for which the Markov property holds i.e. $P_{i j}=P\left(X_{i+1}=j \mid X_{0}=i_{0}, X_{1}=i_{1}, \ldots \ldots . . X_{t}=i\right)=P\left(X_{i+1}=j \mid X_{t}\right)$

If the transition probabilities do not depend on $t$, the Markov chain is said to be stationary.A Markov decision process (MDP) is an extension of a Markov chain, where the Markov chain can be steered by actions and with which optimal actions can be determined.

For a Markov chain, the conditional distribution of any future state $X_{n+1}$ given the past states $X_{0}, X_{1}, \ldots \ldots \ldots . . ., X_{n-1}$ and present state $X_{n}$, is independent of the past states and depends only on the present state. The value $P_{i j}$ represents the probability that the process will, when in state $i$, next make a transition into state $j$. Since probabilities are nonnegative and since the process must make a transition into some state, we have that

$$
P_{i j} \geq 0, i, j \geq 0 ; \sum_{j=0}^{\infty} P_{i j}=1, i=0,1, \ldots
$$

Let $\mathrm{P}$ denote the matrix of the transition probabilities $P_{i j}$, so that

$$
\mathrm{P}=\left[\begin{array}{llc}
p_{11} & p_{12} & \ldots . . p_{1 m} \\
\cdot & \cdot & \cdot \\
\cdot & \cdot & \cdot \\
p_{m 1} & p_{m 2} & \ldots . . p_{m m}
\end{array}\right]
$$




\subsection{Markov Processes:}

A stochastic process $X=\left(X_{n} ; n \geq 0\right)$ with values in a set $\mathrm{E}$ is said to be a discrete time Markov process if for every $n \geq 0$ and every set values $x_{0}, \ldots, x_{n} \in E$, we have

$$
P\left(X_{n+1} \in A \mid X_{0}=x_{0}, X_{1}=x_{1}, \ldots, X_{n}=x_{n}\right)=P\left(X_{n+1} \in A \mid X_{n}=x_{n}\right),
$$

Whenever, $\mathrm{A}$ is a subset of $\mathrm{E}$ such that $\left\{X_{n+1} \in A\right\}$ is an event. In this case, the functions defined by

$$
P_{n}(x, A)=P\left(X_{n+1} \in A \mid X_{n}=x\right)
$$

are called the one - step transition probabilities of X.

Transition probabilities - the probability of moving from one state to another. one-step transition probabilities -The one-step transition probability is the probability that the process, when in state $i$ at time $\mathrm{n}$, will next made a transition to state $\mathrm{j}$ at time $\mathrm{n}+1$. We write

$p_{i j}^{n, n+1}=P\left(X_{n+1}=j \mid X_{n}=i\right)$

i) $\quad 0 \leq p_{i j}^{(n, n+1)} \leq 1$ Since the transition probabilities are (conditional) probabilities.

ii) $\quad \sum_{j=0}^{\infty} p_{i j}^{(n, n+1)}=1$ Since the chain must transition somewhere and summing over all $\mathrm{j}$ is an application of the addition law for a set of disjoint and exhaustive events.

\section{Literature Review}

Markov chain, a well-known subject introduced by Markov in 1906, has been studied by a host of researchers for many years (Chung, 1960; Doob, 1953; Feller, 1971; Kushner \& Yin, 1997). Markovian formulations (see Chiang, 1980; Taylor \& Karlin, 1998; Yang, Yin, \& Zhang, 2002; Yang, \& Yin,2001; Yin \& Zhang, 1997, 1998; Yin, \& Zhang,1995) are useful in solving a number of real-world problems under uncertainties such as determining the inventory levels for retailers, maintenance scheduling for manufacturers, and scheduling and planning in production management.

Markov chain approach has been applied in the design, optimization, and control of queuing systems, manufacturing processes, reliability studies and communication networks, where the underlying system is formulated as stochastic control problem driven by Markovian noise.

In the area of integrated procurement-production systems, Golhar and Sarker (1992), Jamal and Sarker (1993), and Sarker and Parija (1994) implemented various solution methodologies for the integrated model and determined an optimal or near-optimal ordering policy for procurement of raw materials and the manufacturing batch size to minimize the total cost while considering equal shipments of the finished products, at fixed intervals, to the buyers.

Golhar and Sarker (1992) developed the solution methodology for this model using a one-directional search procedure to obtain an optimal or near optimal solution iteratively. However, their procedure finds the break points (shipment points) only when the production rate is equal to the demand rate of finished goods inventory, and this is not the case for all the time. Later, their procedure was improved by Jamal and Sarker (1993) in order to get the break points at each iteration when the production rate is also greater than the demand rate of finished goods inventory.

Furthermore, integrated inventory models implemented by Lu (1995), Goyal (1995), Hill (1997), Hill (1999) and Goyal and Nebebe (2000) cover integrated vendor buyer systems without taking the raw material procurement into consideration.

$\mathrm{Lu}$ (1995) developed an optimal policy for a single-vendor single-buyer problem in which the delivery quantity to the buyer is identical at each replenishment. Then Goyal (1995) and Hill (1997) removed the restriction of identical shipments and allows delivering all available vendor inventories to the buyer. Their models showed that 'deliver what is produced' is better than 'identical delivery quantity'.

However, Viswanathan (1998) discussed that none of the strategies explained by Lu (1995), Goyal (1995) and Hill (1997) obtains the best results for all possible problem parameters. Hill (1999) and Goyal and Nebebe (2000) kept working on IVB systems to obtain a better optimal solution while considering alternative policies.

More recently, Lee (1995) proposed an integrated inventory model for a single-manufacturer singlebuyer supply chain problem by combining IVB (Integrated Vendor Buyer) and IPP (Integrated Procurement Production) systems together. Therefore, the joint economic lot sizes of manufacturer's raw material ordering, manufacturing batch, and buyer's ordering are generated by the developed model. 


\section{Mathematical Methodologies}

4.1 Model Equations And Theorems

4.1.1 Theorem (Chapman-Kolmogorov Equations):

Assume that $\mathrm{X}$ is a time-homogeneous discrete time Markov chain (DTMC)

with n-step transition probabilities $p_{i j}{ }^{(n)}$. Then, for any non-negative integer

$\mathrm{r}<\mathrm{n}$, the identities

$$
p_{i j}{ }^{(n)}=\sum_{k \in E} p_{i k}{ }^{(r)} p_{k j}{ }^{(n-r)}
$$

hold for all $i, j \in E$.

Proof:

By using first law of total probability and then the Markov property, we have

$p_{i j}{ }^{(n)}=p\left\{X_{n}=j \mid X_{0}=i\right\}$

$=\sum_{k \in E} p\left(X_{n}=j, X_{r}=k \mid X_{0}=i\right)$

$=\sum_{k \in E} p\left(X_{n}=j, X_{r}=k \mid X_{0}=i\right) \cdot p\left(X_{r}=k \mid X_{0}=i\right)$

$$
\begin{aligned}
& =\sum_{k \in E} p\left(X_{n}=j, X_{r}=k\right) \cdot p\left(X_{r}=k \mid X_{0}=i\right) \\
& =\sum_{k \in E} p_{i k}{ }^{(r)} p_{k j}{ }^{(n-r)}
\end{aligned}
$$

\subsubsection{Definition ( Optimality Equations)}

Given a finite-horizon Markov decision problem with decision epochs $T=\{1, \ldots, N\}$, defined on the optimal value functions $u_{t}^{*}: H_{t} \rightarrow R$ such that

$$
u_{t}^{*}\left(h_{t}\right)=\sup _{\pi \in \prod H R} u_{t}^{\pi}\left(h_{t}\right)
$$

Where $u_{t}^{\pi}\left(h_{t}\right)$ is the expected total reward earned by using policy $\pi$ from time $\mathrm{t}$ to $\mathrm{N}$ and the supremum is taken over all history-dependent randomized policies. The optimality equation is:

$$
u_{t}\left(h_{t}\right)=\sup _{a \in A_{s_{t}}}\left\{r_{t}\left(s_{t}, a\right)+\sum_{j \in s} p_{t}\left(j \mid s_{t}, a\right) u_{t+1}\left(h_{t}, a, j\right)\right\}
$$

for $t=1, \ldots, N-1$ and $h_{t}=\left(h_{t-1}, a_{t-1,} s_{t}\right)$ along with the boundary condition

$$
u_{N}\left(h_{N}\right) \equiv r_{N}\left(s_{N}\right)
$$

for $h_{N}=\left(h_{N-1}, a_{N-1}, s_{N}\right)$. The supremum in equation (8.0) is taken over the set of all possible actions that are available when the system is in state $s_{t}$ and this can be replaced by a maximum when all of the action sets are finite.

\section{4..1.3 Development of Value Iteration Method}

Let $X_{n}$ denote the state of the process at time $\mathrm{n}$ and $a_{n}$ the action chosen at time $\mathrm{n}$, then the above is equivalent to stating that: 


$$
P\left(X_{n+1}=j \mid X_{0}, a_{0}, X_{1}, a_{1}, \ldots \ldots . . X_{n}=i, a_{n}=a\right)=P_{i j}(a)
$$

We consider an aperiodic irreducible Markov chain with $m$ states

$(\mathrm{m}<\infty)$ and the transition probability matrix $\mathrm{P}$ with every transition, $\mathrm{i}$ to $\mathrm{j}$ associate a reward $R_{i j}$ if we let $V_{i}^{(n)}$ be the expected total earnings (reward) in the next $\mathrm{n}$ transitions, given that the system is in state $\mathrm{i}$ at present.

A simple relation can be given

For $\left\{V_{i}^{(n)}\right\}_{n-1}^{\infty}$ as follows:

$$
V_{i}^{(n)}=\sum_{j=1}^{m} P_{i j}\left[R_{i j}+V_{j}^{(n-1)}\right] i=1,2, \ldots \ldots \ldots, m ; n=1,2,3, \ldots \ldots \ldots
$$

Let $\quad \sum_{j=1}^{m} P_{i j} R_{i j}=Q_{i}$

Equation (11)

can now be written as:

$$
V_{i}^{(n)}=Q_{i}+\sum_{j=1}^{m} P_{i j} V_{j}^{(n-1)}
$$

... we get

$$
\begin{aligned}
V_{i}^{(1)}= & Q_{i}+\sum_{j-1}^{m} P_{i j} V^{(0)} \\
& V_{i}^{(2)}=Q_{i}+\sum_{j=1}^{m} P_{i j}\left[Q_{j}+\sum_{k=1}^{m} P_{j k} V_{k}^{(0)}\right]=Q_{i}+\sum_{j=1}^{m} P_{i j} Q_{j}+\sum_{k=1}^{m} \sum_{j=1}^{m} P_{i j} P_{j k} V_{k}^{(0)} \\
= & Q_{i}+\sum_{j=1}^{m} P_{i j} Q_{j}+\sum_{k=1}^{m} \sum_{j=1}^{m} P_{i j} P_{j k} V_{k}^{(0)}=Q_{i}+\sum_{j=1}^{m} P_{i j} Q_{j}+\sum_{k=1}^{m} P_{i k}^{(2)} V_{k}^{(0)}
\end{aligned}
$$

Setting $\mathrm{n}=1,2$

Where $P_{i j}^{(n)}$ is the $(i, j)^{\text {th }}$ element of the matrix $P^{n}$

Let $V^{(n)}=\left[\begin{array}{l}V_{1}^{(n)} \\ V_{2}^{(n)} \\ V_{3}^{(n)} \\ \cdot \\ \cdot \\ V_{m}^{(n)}\end{array}\right] Q=\left[\begin{array}{l}Q_{1} \\ Q_{2} \\ Q_{3} \\ \cdot \\ \cdot \\ Q_{m}\end{array}\right]$

Equation (13) can be put in matrix notation as

$$
V^{(n)}=Q+P Q+P^{2} V^{(0)}
$$

Extending this to a nth term, we have

$$
V^{(n)}=Q+P Q+P^{2} Q+\ldots \ldots+P^{(n-1)} Q+P^{n} V^{(0)}=\left[1+\sum_{k-1}^{n-1} P K\right] Q+P^{n} V^{(0)}
$$

\subsubsection{Value Iteration ( algorithm)}

The value iteration algorithm is a method that can be used to find $\varepsilon$-optimal policies for discounted Markov decision processes. The algorithm consists of the following steps:

- Set $n=0$ and choose an error tolerance $\varepsilon>0$ and an initial condition $v^{0} \in V$

- For each $s \in S$, compute $V^{n+1}(s)$,where; 


$$
V^{n+1}(s)=\max _{a \in A_{s}}\left\{r\left(s, a+\lambda \sum_{j \in s} p(j \mid s, a) v^{(n)}(j)\right\}\right.
$$

- If

$$
\square v^{n+1}-v^{n} \square<\frac{\varepsilon(1-\lambda)}{2 \lambda},
$$

, go to step 4. Otherwise increase $n$ to $n+1$ and return to

step 2.

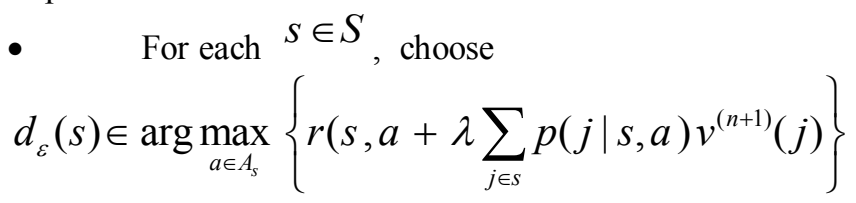

And stop.

In vector notation, this algorithm can be expressed as:

\subsection{Model Formulation}

$$
\begin{aligned}
& v^{n+1}=L v^{n} \\
& d_{\varepsilon} \in \arg \max _{d \in D}\left\{r d+\lambda P_{d} v^{n+1}\right\}
\end{aligned}
$$

We consider a manufacturer of a certain product who has found tough competition in business and would like to use analytical techniques in making decisions for advertising, investing in research and development of new products: using months as the time unit, the sales of product undergos state changes between rising (1), steady (2) and dropping (3) states based on the following transition probability and reward matrix.

$$
\begin{gathered}
\mathrm{P}=\left[\begin{array}{lll}
p_{11} & p_{12} & \ldots . . p_{1 m} \\
\cdot & \cdot & \cdot \\
\cdot & \cdot & \cdot \\
p_{m 1} & p_{m 2} & \ldots . . p_{m m}
\end{array}\right] \\
R=\left[\begin{array}{ccc}
R_{11} & R_{12} & \ldots . R_{1 m} \\
. . & . . & \ldots . . \\
R_{m 1} & R_{m 2} & \ldots . R_{m m}
\end{array}\right]
\end{gathered}
$$

Let the position of the sales of the product be described by a random variable $(\mathrm{X})$, suppose that the sales is considered for several months; (n), we obtain a stochastic process $X_{n} n=1,2,3, \ldots$ we assume that the position of the sales are:

(1) Rising sales (state1)

(2) Steady sales (state 2)

(3) Drop in sales (state 3)

We consider the states to be mutually exclusive and exhaustive. It is further assumed that the stochastic process $X_{n}, n=1,2,3, \ldots .$. is governed by a first order Markov chain mentioned in equation (1.0). The possible transitions between the states are presented in figure (1).

From the transition diagram in figure 1 and equation (1.0) where $m, n=1,2,3$. We obtain a transition matrix $P$. We assume that the matrix is $\mathrm{P}$ is aperiodic, irreducible stochastic matrix.

When the sales of product is in state 1 , let there be two alternatives open to the manufacturer:

Alternative 1: continue without change

Alternative 2: increase advertising

Let the corresponding transition probabilities and rewards be given as:

$\left[\begin{array}{lll}{ }^{1} P_{11} & { }^{1} P_{12} & { }^{1} P_{13}\end{array}\right]$ 
$\left[\begin{array}{lll}{ }^{1} R_{11} & { }^{1} R_{12} & { }^{1} R_{13}\end{array}\right]$

And

$\left[{ }^{2} P_{11}{ }^{2} P_{12} \quad{ }^{2} P_{13}\right]$

$\left[\begin{array}{lll}{ }^{2} R_{11} & { }^{2} R_{12} & { }^{2} R_{13}\end{array}\right]$ respectively.

When the sales of the product is in state 2, let the alternatives be as:

Alternative 1: continue without change

Alternative 2: invest in research.

Suppose that the corresponding transition probability and rewards are given as:

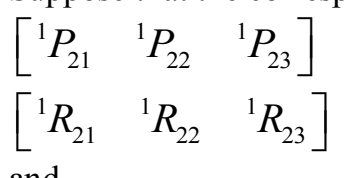

and

$$
\begin{aligned}
& {\left[\begin{array}{lll}
{ }^{2} P_{21} & { }^{2} P_{22} & { }^{2} P_{23}
\end{array}\right]} \\
& {\left[\begin{array}{lll}
{ }^{2} R_{21} & { }^{2} R_{22} & { }^{2} R_{23}
\end{array}\right] \text { respectively. }}
\end{aligned}
$$

When the sales of the product is in state 3 , let the following alternatives be open to him.

Alternative 1: change

Alternative 2: development of new product.

and

$$
\begin{aligned}
& {\left[{ }^{1} P_{31}{ }^{1} P_{32}{ }^{1} P_{33}\right]} \\
& {\left[\begin{array}{lll}
{ }^{1} R_{31} & { }^{1} R_{32} & { }^{1} R_{33}
\end{array}\right]}
\end{aligned}
$$

$$
\begin{aligned}
& {\left[\begin{array}{lll}
{ }^{2} P_{31} & { }^{2} P_{32} & { }^{2} P_{33}
\end{array}\right]} \\
& {\left[\begin{array}{lll}
{ }^{2} R_{31} & { }^{2} R_{32} & { }^{2} R_{33}
\end{array}\right]}
\end{aligned}
$$

respectively.

\section{Application}

A manufacturer of certain a product has found tough competition in business and would like to use analytical techniques in making decisions for advertising, investing in research and development of new products. The product undergoes state changes between rising sales, steady sales and drop in sales based on the following transition matrices and corresponding reward matrices.

Let the transition probabilities $\left(P_{i j}\right)$ and the corresponding reward $\left(R_{i j}\right)$ be given as follows:

$$
P=P_{i j}=\left(\begin{array}{lll}
P_{11} & P_{12} & P_{13} \\
P_{21} & P_{22} & P_{23} \\
P_{31} & P_{32} & P_{33}
\end{array}\right) ; i, j=1,2,3
$$

and

$$
R=R_{i j}=\left(\begin{array}{lll}
R_{11} & R_{12} & R_{13} \\
R_{21} & R_{22} & R_{23} \\
R_{31} & R_{32} & R_{33}
\end{array}\right) ; i, j=1,2,3
$$

Let $\mathrm{D}$ be the decision set and we have two alternative decisions available to the manufacturer. That is, Alternative 1; and Alternative 2; Thus in every state we have $\mathrm{k}=1,2 \in \mathrm{D}$.

Suppose we have the following transition probabilities and the corresponding reward matrices.

For $\mathrm{k}=1$ 


$$
\begin{aligned}
& P=\left(\begin{array}{ccc}
{ }^{1} p_{11} & { }^{1} p_{12} & { }^{1} p_{13} \\
{ }^{1} p_{21} & { }^{1} p_{22} & { }^{1} p_{23} \\
{ }^{1} p_{31} & { }^{1} p_{32} & { }^{1} p_{33}
\end{array}\right)=\left(\begin{array}{ccc}
0.6 & 0.2 & 0.2 \\
0.1 & 0.6 & 0.3 \\
0.3 & 0.5 & 0.2
\end{array}\right) \\
& R=\left(\begin{array}{ccc}
{ }^{1} R_{11} & { }^{1} R_{12} & { }^{1} R_{13} \\
{ }^{1} R_{21} & { }^{1} R_{22} & { }^{1} R_{23} \\
{ }^{1} R_{31} & { }^{1} R_{32} & { }^{1} R_{33}
\end{array}\right)=\left(\begin{array}{ccc}
6 & 5 & 4 \\
4 & 3 & -2 \\
4 & -6 & 3
\end{array}\right)
\end{aligned}
$$

For $\mathrm{k}=2$

$$
\begin{aligned}
& P=\left(\begin{array}{lll}
{ }^{2} p_{11} & { }^{2} p_{12} & { }^{2} p_{13} \\
{ }^{2} p_{21} & { }^{2} p_{22} & { }^{2} p_{23} \\
{ }^{2} p_{31} & { }^{2} p_{32} & { }^{2} p_{33}
\end{array}\right)=\left(\begin{array}{ccc}
0.6 & 0.1 & 0.3 \\
0.5 & 0.3 & 0.2 \\
0.1 & 0.3 & 0.6
\end{array}\right) \\
& R=\left(\begin{array}{lll}
{ }^{2} R_{11} & { }^{2} R_{12} & { }^{2} R_{13} \\
{ }^{2} R_{21} & { }^{2} R_{22} & { }^{2} R_{23} \\
{ }^{2} R_{31} & { }^{2} R_{32} & { }^{2} R_{33}
\end{array}\right)=\left(\begin{array}{ccc}
6 & 3 & 2 \\
-2 & -10 & 5 \\
-7 & -8 & -1
\end{array}\right)
\end{aligned}
$$

VI. Results

We shall use these values in the equation below to determine the best policies for every $\mathrm{n}$

$$
{ }^{o} V_{i}^{(n)}=\max _{k \in D}\left[{ }^{k} Q_{i}+\sum_{j=1}^{m}{ }^{k} P_{i j}{ }^{o} V_{j}^{(n-1)}\right] n=1,2, \ldots . ; i=1,2, \ldots . ., m .
$$

we have

The summary of the results is presented in Table 1 .

Table 1: The summary result of the optimal policies and rewards

\begin{tabular}{|l|l|l|l|l|l|l|}
\hline $\mathrm{N}$ & $d_{1}^{(n)}$ & $d_{2}^{(n)}$ & $d_{3}^{(n)}$ & ${ }^{o} V_{1}^{(n)}$ & ${ }^{o} V_{2}^{(n)}$ & ${ }^{o} V_{3}^{(n)}$ \\
\hline 1 & 1 & 1 & 1 & 540 & 160 & -120 \\
2 & 1 & 1 & 1 & 872 & 274 & -8.2 \\
3 & 1 & 2 & 1 & $1,433.6$ & 675.8 & 300.2 \\
4 & 1 & 2 & 1 & $2,488.96$ & $1,655.38$ & $1,128.22$ \\
5 & 1 & 2 & 1 & $4,539.06$ & $3,622.12$ & $2,928.24$ \\
6 & 1 & 2 & 1 & $8,572.57$ & $7,563.93$ & $6,686.67$ \\
\hline
\end{tabular}

\subsection{Discussion Of Results}

The results indicate the best policies for each n. $d_{i}^{(n)}$ where $n=1,2,3,4,5,6$ and $i=1,2,3$. Thus, we have obtained the best policies for the three states for six months. In addition to the best policies, the corresponding expected rewards are also provided.

For the first month, $d_{1}^{(1)}=1$ with ${ }^{o} V_{1}^{(1)}=540$ means that the best policy for state 1 is for the manufacturer to continue without changing the method and the corresponding expected reward is five hundred and forty thousand naira and the same results follow for all the subsequent iterations

The results revealed that for the fourth, fifth and sixth month, the best policies for the states is alternative 1 while for the first state, Alternative 2 for the second state and Alternative 1 for the third state respectively. This is a convergence to stable policy that further iterations beyond is not necessary.

\section{Conclusion}

From the results obtained, manufacturing industries should all take the advertisement very seriously and should always involve in research to improve the level of their production which will therefore increase the sales to maximize profits.

\section{References}


[1]. Bellman, R. (1957). “A Markovian Decision Process.” J. Math. Mech. 6. 679-684.

[2]. Bellman, R. (1957). Dynamic Programming Princeton: Princeton University Press.

[3]. Chung, K. L. (1960). Markov chain with stationary transition probabilities. Berlin: Springer.

[4]. Doob, J. L. (1953). Stochastic processes. New York: Wiley.

[5]. Derman, C. (1970). Finite State Markovian Decision Processes. New York Academic Press.

[6]. Feller, W. (1971). An introduction to probability theory and its applications. New York: Wiley.

[7]. Golhar, D.Y. and Sarker, B.R. (1992), "Economic manufacturing quantity in a just-in-time delivery system,” International Journal of Production Research, 30(5): 961-972.

[8]. Goyal, S.K. and Nebebe, F (2000), "Determination or economic production- shipment policy for a single-vendor single buyer system,” European Journal of Operational Research, 121(1): 175-178

[9]. Heyman, D.P., and Sobel, M.J. (1983). Stochastic Models I Operations Research. Vol.2. New York: McGraw-Hill.

[10]. Hill, R.M. (1997), "The single-vendor single-buyer integrated production-inventory model with a generalized policy," European Journal of Operational Research, 97(3): 493-499.

[11]. Howard RA. Dynamic programming and Markov Processes; Technology Press of MIT.1960.

[12]. Howard, R.A. (1971). Dynamic Probabilistic Systems. Vols. 1, 2. New York: Willey.

[13]. Kushner, H. J., \& Yin, G. G. (1997). Stochastic approximation algorithms and applications. New York: Springer.

[14]. Lambert, D.M. and Cooper, M.C. (2000), "Issues in supply chain management," Industrial Marketing Management, 29(1): 65-83.

[15]. Lovejoy WS. A survey of algorithmic methods for partially observed Markov decision processes. Annuals of Operations Research 1991 28: 47-66.

[16]. Markov Decision Processes: Lecture Notes for STP 425 by Jay Taylor, November 25, 2012.

[17]. Miller, B.L. (1968). "Finite State Continuous Time Markov Decision Processes with a Finite Planning Horizon." SIAM J. Control 6, 266-280.

[18]. Monahan, GE. A survey of partially observable Markov decision processes: theory, models and algorithms. Management Science 1982 28(1): 1-16.

[19]. Puterman, M. 1994. Markov Decision Processes: Discrete Stochastic Dynamic Programming. John Wiley and Sons, New York, NY.

[20]. Ross, S.M. (1983). Introduction to stochastic dynamic programming. New York: Academic Press.

[21]. Sobel, M. J., R. Q. Zhang. 2001. Inventory policies for systems with stochastic and deterministic demand. Operations Research 49, $157-162$

[22]. Song, J. S., P. Zipkin. 2003. Supply chain operations: Assemble-to-order systems.

[23]. Taylor, H. M., \& Karlin, S. (1998). An introduction to stochastic modeling. Boston: Academic Press.

[24]. Van Mieghem, J. A., N. Rudi. 2001. Newsvendor networks: Inventory management and capacity investment with discretionary activities. Manufacturing and Service Operations Management 4, 313-335.

[25]. Veinott, A.F., Jr. (1966). “The Status of Mathematical Inventory Theory.” Management sci. 12, 745-777.

[26]. White DJ, (1963), Dynamic programming, Markov chains, the method of successive approximations, Journal of Mathematical Analysis and Applications.

[27]. Yin, G. G., \& Zhang, Q. (1997). Mathematics of stochastic manufacturing systems. Providence: American Mathematical Society. 